\title{
Clinical course and outcomes of COVID-19 in rheumatic disease patients: a case cohort study with a diverse population
}

\author{
Timothy Arleo ${ }^{1} \cdot$ David Tong $^{2} \cdot$ Julie Shabto $^{1} \cdot$ Ghazala O'Keefe $^{3} \cdot$ Arezou Khosroshahi $^{4}$ \\ Received: 9 December 2020 / Revised: 28 December 2020 / Accepted: 1 January 2021 / Published online: 9 January 2021 \\ (C) International League of Associations for Rheumatology (ILAR) 2021
}

\begin{abstract}
Objective To determine clinical course and outcomes in rheumatic disease patients with coronavirus disease 2019 (COVID-19) and compare results to uninfected patients.

Methods We conducted a case cohort study of autoimmune disease patients with COVID-19 (confirmed by severe acute respiratory syndrome coronavirus 2 PCR) from February 1, 2020, to July 31, 2020, and compared them in a 1:3 ratio with uninfected patients who were matched based on race, age, sex, and comorbidity index. Patient demographics, clinical course, and outcomes were compared among these patient groups.

Results A total of 70 rheumatic disease patients with COVID-19 (mean age, 56.6 years; 64\% African American) were identified. The $34(49 \%)$ patients who were hospitalized used oral glucocorticoids more frequently than those treated as outpatients $(p<0.01)$. All 10 patients using anti-TNF $\alpha$ medications were treated as outpatients $(p<0.01)$. Those hospitalized with COVID-19 more often required ICU admission (17 (50\%) vs $27(26 \%), p=0.01)$ and intubation (10 (29\%) vs $6(6 \%)$, $p<0.01)$ than uninfected patients and had higher mortality rates $(6(18 \%)$ vs $3(3 \%), p<0.01)$. Of the six COVID-19 patients who died, only one was of African ancestry $(p=0.03)$.

Conclusion Rheumatic disease patients infected with COVID-19 were more likely to require ICU admission, ventilation, and died more frequently versus uninfected patients with autoimmune disease. Patients on anti-TNF $\alpha$ medications were hospitalized less frequently, while those on chronic glucocorticoids were hospitalized more frequently. These findings have important implications for medication choice in rheumatic disease patients during the ongoing spread of COVID-19.

\section{Key Points}

- We show that hospitalized rheumatic disease patients with COVID-19 have poorer outcomes including ICU admission, ventilation, and death compared to hospitalized rheumatic disease patients not infected with COVID-19.

- This study adds further support regarding protective effects of anti-TNF $\alpha$ medications in COVID-19 disease course, with 0 of 10 of these patients required hospitalization.
\end{abstract}

Keywords Immunosuppressive agents $\cdot$ Rheumatic diseases · SARS-CoV-2 $\cdot$ Tumor necrosis factor inhibitors

Arezou Khosroshahi

akhosroshahi@emory.edu

1 Emory University School of Medicine, Atlanta, GA, USA

2 Division of Hospital Medicine, Emory University School of Medicine, Atlanta, GA, USA

3 Department of Ophthalmology, Emory University School of Medicine, Atlanta, GA, USA

4 Division of Rheumatology, Emory University School of Medicine, 244 Whitehead Bldg., 615 Michael Street, Atlanta, GA 30322, USA

\section{Introduction}

Data on COVID-19 in patients with immune-mediated rheumatic diseases using immunosuppressive medications such as biologic and conventional disease-modifying anti-rheumatic drugs (DMARDs), chronic glucocorticoids, and others is limited. Recent literature suggests that DMARDs may provide protective effects by disrupting the cytokine storm reaction that leads to serious disease $[1,2]$, while analysis of chronic glucocorticoid use shows increased rates of hospitalization from COVID-19 due to compromised immunity [3]. We aim to leverage our large rheumatic disease population with high 
prevalence of African American patients infected with COVID-19 to further characterize outcomes based on immunosuppressive medication.

Patients with rheumatic disease on immunosuppressive medications are considered at higher risk for contracting severe COVID-19 disease [3-5]. A potential confounder is that uninfected, admitted rheumatic disease patients experience poor outcomes regardless of COVID-19 infection, with up to $1 / 3$ requiring ICU admission $[6,7]$. We aim to further characterize the impact of COVID-19 infection by comparing infected, admitted COVID-19 patients to uninfected, admitted patients.

\section{Methods}

\section{Study design}

We conducted a case cohort study on rheumatic disease patients with a positive PCR test result for COVID-19 through the Emory Healthcare System using a data warehouse search. Patients tested between February 1, 2020 and July 31, 2020, were considered. PCR-positive patients were compared to matched control patients in a 1:3 ratio and were matched based on sex, age, race, and Elixhauser comorbidity index. Matches were prioritized based on Elixhauser score, followed by age. More detailed methodology can be found in Online Resource 1.

\section{Selection criteria}

Patients were considered if they had a diagnosed autoimmune condition and were on chronic immunosuppressants. Search criteria were based on a standardized list of autoimmune diseases (Online Resource 2) and immunosuppressive medications (Online Resource 3). Diagnoses were confirmed by study authors. Control patients were selected among hospitalized patients with no clinical concern for COVID-19 or who received a negative PCR test. Patients were excluded if they received a transplanted organ or were hospitalized for elective procedures or psychiatric conditions.

\section{Main outcome variable}

Variables were extracted from the EHR using manual review. Main outcome variables included hospitalization status, immunosuppressive medications, and outcomes among those hospitalized, including hospitalization length, ICU admission, ventilator use, discharge disposition, and death. Data was also collected on demographics, primary rheumatic disease, medical comorbidities, symptoms at time of PCR test date or admission, and peak laboratory values among those hospitalized.

\section{Statistical analysis}

Continuous variables were analyzed using a two-tailed Welch $t$ test assuming unequal variance and are presented as mean ( $95 \% \mathrm{CI})$. Categorical variables were analyzed using a 2-tailed Fisher test and are presented as number (percentage).

\section{Results}

From February 1, 2020, to July 31, 2020, 6536 patients tested positive for COVID-19. Of these, seventy had been diagnosed with rheumatic disease with immunosuppressive medication treatment (Table 1). The most common autoimmune diagnoses included rheumatoid arthritis (26, 37\%), systemic lupus erythematosus (SLE) $(8,11 \%)$, polymyalgia rheumatica (PMR) or giant cell arteritis (GCA) $(7,10 \%)$, irritable bowel disease (IBD) $(6,9 \%)$, and sarcoidosis $(5,7 \%)$. Forty-five $(64 \%)$ of the COVID-19-positive patients were African American.

\section{Admitted, COVID-19 versus outpatient, COVID-19 patients}

As seen in Table 1, 34 (49\%) of the COVID-19 patients were hospitalized. Compared to those treated outpatient, sex and race distribution were similar $(p=1.00$ and $p=0.77$, respectively), but hospitalized patients were older with mean ages of 65.2 versus $48.4(p<0.01)$. African American patients were hospitalized at similar rates, with 22 of $45(49 \%)$ being admitted. Hospitalized patients were more likely to have comorbidities of renal disease and congestive heart failure $(p<0.01$ and $p=0.02$, respectively). All seven PMR or GCA patients were hospitalized, while all six IBD patients were treated as outpatients $(p<0.01$ and $p=0.03$, respectively). Hospitalized patients were more likely to receive chronic glucocorticoids and were less likely to take a biologic DMARD (bDMARD) ( $p<0.01$ and $p=0.02$, respectively). Of those using bDMARDs, none of the ten patients receiving anti-TNF $\alpha$ treatment were admitted $(p<0.01)$. The use of hydroxychloroquine (HCQ) and other chronic immunosuppressive medications did not differ between groups. Admitted patients more frequently experienced fever and dyspnea ( $p=0.02$ and $p=0.01$, respectively) as presenting symptoms. Other symptoms did not differ between groups.

\section{Admitted, COVID-19 versus admitted, uninfected patients}

Admitted COVID-19 patients were compared to matched uninfected comparators as seen in Table 2. The distribution of sex, age, and race were similar $(p=1.00, p=0.52$, and $p=0.78$, respectively). The frequency of comorbidities, including the Elixhauser comorbidity indices, was similar between groups. 
Table 1 Baseline characteristics and manifestations in rheumatic disease patients with COVID-19 who were hospitalized ( $n=34)$ versus treated outpatient $(n=36)$

\begin{tabular}{|c|c|c|c|}
\hline Characteristic & $\begin{array}{l}\text { Inpatient COVID-19 } \\
(n=34)\end{array}$ & $\begin{array}{l}\text { Outpatient COVID-19 } \\
(n=36)\end{array}$ & $P$ value \\
\hline Female $n(\%)$ & $27(79 \%)$ & $29(81 \%)$ & 1.00 \\
\hline Age in years, mean $[95 \% \mathrm{CI}]$ & $65.2[59.1-71.2]$ & $48.4[44.1-52.7]$ & 0.00 \\
\hline Race, $n(\%)$ & & & $0.94 *$ \\
\hline Caucasian & $9(26 \%)$ & $11(31 \%)$ & \\
\hline Black or African American & $22(65 \%)$ & $23(64 \%)$ & \\
\hline Other $^{\dagger}$ & $3(9 \%)$ & $2(6 \%)$ & \\
\hline \multicolumn{4}{|l|}{ Comorbidities, $n(\%)$} \\
\hline Pulmonary disease $e^{\ddagger}$ & $8(24 \%)$ & $3(8 \%)$ & 0.11 \\
\hline Diabetes & $10(29 \%)$ & $6(17 \%)$ & 0.26 \\
\hline Renal disease & $12(35 \%)$ & $2(6 \%)$ & 0.00 \\
\hline Cancer & $2(6 \%)$ & $5(14 \%)$ & 0.43 \\
\hline Hypertension & $24(71 \%)$ & $19(53 \%)$ & 0.22 \\
\hline Coronary artery disease & $6(18 \%)$ & $1(3 \%)$ & 0.22 \\
\hline Congestive heart failure & $9(26 \%)$ & $2(6 \%)$ & 0.02 \\
\hline Obesity, BMI 30+ kg/m2 & $13(38 \%)$ & $15(42 \%)$ & 0.81 \\
\hline Smoking & $10(29 \%)$ & $11(31 \%)$ & 1.00 \\
\hline \multicolumn{4}{|l|}{ AI diagnosis, $n(\%)$} \\
\hline RA & $13(38 \%)$ & $13(36 \%)$ & 1.00 \\
\hline SLE & $5(15 \%)$ & $3(8 \%)$ & 0.47 \\
\hline PMR or GCA & $7(21 \%)$ & $0(0 \%)$ & 0.00 \\
\hline IBD & $0(0 \%)$ & $6(17 \%)$ & 0.03 \\
\hline Sarcoidosis & $2(6 \%)$ & $3(8 \%)$ & 1.00 \\
\hline Vasculitis & $2(6 \%)$ & $1(3 \%)$ & 0.61 \\
\hline ILD & $2(6 \%)$ & $0(0 \%)$ & 0.23 \\
\hline Sjogren's syndrome & $1(3 \%)$ & $1(3 \%)$ & 1.00 \\
\hline Mixed SLE and MCTD & $0(0 \%)$ & $2(6 \%)$ & 1.00 \\
\hline Castleman disease/TAFRO & $1(3 \%)$ & $0(0 \%)$ & 1.00 \\
\hline MCTD & $1(3 \%)$ & $0(0 \%)$ & 0.49 \\
\hline Scleroderma & $0(0 \%)$ & $1(3 \%)$ & 1.00 \\
\hline Psoriatic arthritis & $0(0 \%)$ & $1(3 \%)$ & 1.00 \\
\hline Adult-onset still's disease & $0(0 \%)$ & $1(3 \%)$ & 1.00 \\
\hline Autoimmune hepatitis & $0(0 \%)$ & $1(3 \%)$ & 1.00 \\
\hline Antisynthetase syndrome & $0(0 \%)$ & $1(3 \%)$ & 1.00 \\
\hline Spondyloarthropathy & $0(0 \%)$ & $1(3 \%)$ & 1.00 \\
\hline Ankylosing spondylitis & $0(0 \%)$ & $1(3 \%)$ & 1.00 \\
\hline \multicolumn{4}{|l|}{ Medications, $n(\%)$} \\
\hline Oral glucocorticoid & $24(71 \%)$ & $13(36 \%)$ & 0.00 \\
\hline$>10 \mathrm{mg} /$ day & $14(41 \%)$ & $7(19 \%)$ & 0.07 \\
\hline Hydroxychloroquine & $10(29 \%)$ & $10(28 \%)$ & 1.00 \\
\hline bDMARDs & $3(9 \%)$ & $12(33 \%)$ & 0.02 \\
\hline anti-TNF $\alpha$ & $0(0 \%)$ & $10(28 \%)$ & 0.00 \\
\hline Rituximab & $3(9 \%)$ & $2(6 \%)$ & 0.67 \\
\hline csDMARDs & $12(35 \%)$ & $15(42 \%)$ & 0.63 \\
\hline Methotrexate & $5(15 \%)$ & $7(19 \%)$ & 0.75 \\
\hline Mycophenolate mofetil & $4(12 \%)$ & $1(3 \%)$ & 0.19 \\
\hline Leflunomide & $2(6 \%)$ & $0(0 \%)$ & 0.23 \\
\hline Azathioprine & $1(3 \%)$ & $7(19 \%)$ & 0.06 \\
\hline
\end{tabular}


Table 1 (continued)

\begin{tabular}{llll}
\hline Characteristic & $\begin{array}{l}\text { Inpatient COVID-19 } \\
(n=34)\end{array}$ & $\begin{array}{l}\text { Outpatient COVID-19 } \\
(n=36)\end{array}$ \\
\hline tsDMARDs & $0(0 \%)$ & $1(3 \%)$ & 1.00 \\
Tofacitinib & $0(0 \%)$ & $1(3 \%)$ & 1.00 \\
Cyclophosphamide & $1(3 \%)$ & $0(0 \%)$ & 0.49 \\
Sirolimus & $1(3 \%)$ & $0(0 \%)$ & 0.49 \\
Presenting symptoms, $n(\%)$ & & $19(53 \%)$ & 0.02 \\
Fever & $25(74 \%)$ & $27(75 \%)$ & 0.78 \\
Cough & $24(71 \%)$ & $8(22 \%)$ & $17(47 \%)$ \\
Dyspnea & $18(53 \%)$ & $8(22 \%)$ \\
Myalgias & $10(29 \%)$ & $14(39 \%)$ \\
Diarrhea & $9(26 \%)$ & 0.01 \\
Loss of taste or smell & $5(15 \%)$ & 0.23 \\
\hline
\end{tabular}

* $P$ value for Race was computed by merging "Caucasian" and "Other" race categories and comparing to "Black or African American"

$\dagger$ "Other" race includes American Indian or Alaska Native, Native Hawaiian or other Pacific Islander and not reported

ॠ Pulmonary disease includes asthma or COPD

${ }^{\S} R A$, rheumatoid arthritis; SLE, systemic lupus erythematosus; $P M R$, polymyalgia rheumatica; $G C A$, giant cell arteritis; $I B D$, irritable bowel disease; $I L D$, interstitial lung disease; $M C T D$, mixed connective tissue disease; $b D M A R D s$, biologic disease modifying anti-rheumatic drugs; $T N F$, tumor necrosis factor; $c s D M A R D s$, conventional synthetic disease modifying anti-rheumatic drugs; tsDMARDs, targeted synthetic disease modifying antirheumatic drugs

There were more PMR or GCA patients in the COVID-19 group $(p=0.04)$, but there were no other differences in frequency in autoimmune diagnoses between groups. Uninfected patients more frequently received HCQ $(p=0.03)$, but medication use was otherwise similar between the groups. COVID-19 patients more frequently experienced fever, cough, myalgias, and loss of taste or smell versus their comparators ( $p<0.01$ for all). As seen in Table 2, frequency of dyspnea was similar $(p=0.47)$. COVID-19 patients had higher maximum C-reactive protein (CRP) values and lower minimum albumin values during their first hospitalization $(p<0.01$ and $p=0.02$, respectively), while other laboratory values were similar between groups.

Patients infected with COVID-19 had longer hospital stays of 12.9 days versus 8.0 days $(p=0.03)$. Admitted COVID-19 patients required ICU admission (17 (50\%) vs $27(26 \%), p=0.01)$ and intubation $(10(29 \%)$ vs $6(6 \%), p<0.01)$ at higher frequencies. Admitted COVID-19 patients remained on ventilators for 12.9 days versus 4.3 days for uninfected patients $(p=0.02)$. COVID-19 patients were less likely to be discharged under self-care $(14(41 \%)$ vs $69(68 \%), p<0.01)$ and were more likely to die while hospitalized (6 (18\%) vs $3(3 \%), p<0.01)$ (Table 2$)$.

\section{Admitted COVID-19: patients who died versus patients who survived}

Six of the 70 COVID-19 patients died, resulting in a $9 \%$ mortality rate. The mortality rate among hospitalized COVID-19 patients was $18 \%$. The distribution of sex was similar between patients who died and survived, but patients who died were older with a mean age of 76.5 vs 62.8 $(p<0.01)$ (Table 3). Hospitalized patients who died were less likely to be African American, with one of 22 African American patients dying, three of nine Caucasian patients dying, and two of three patients of another race dying $(p=0.03)$. There were no differences in comorbid medical conditions between those who died and survived. Of the six patients who died, three had rheumatoid arthritis, two had PMR or GCA, and one had interstitial lung disease secondary to connective tissue disease. Autoimmune disease frequency was similar to surviving patients. All patients who died used glucocorticoids, with five on doses of $10 \mathrm{mg} /$ day or greater. One patient who died took rituximab, while one other patient took mycophenolate mofetil. As seen in Table 3, reported symptom frequency and laboratory values were similar between groups. All six patients who died were admitted to the ICU with four requiring ventilation ( $p<0.01$ and $p=0.03$, respectively).

\section{Discussion}

In this study, we evaluated patients with rheumatic conditions on chronic immunosuppressive medications who developed COVID-19 infections.

Among COVID-19 patients, those hospitalized were more likely to be older, have heart failure, and have renal disease. These are known risk factors for severe COVID-19 outcomes and are supported in other studies [3-5, 8, 9]. Gianfrancesco et al.'s study found additional risk factors including 
Table 2 Baseline characteristics and outcomes in rheumatic disease patients with COVID-19 ( $n=34)$ and age, sex, race, and comorbidity index matched rheumatic disease comparators without COVID-19 ( $n=102)$

\begin{tabular}{|c|c|c|c|}
\hline Characteristics & $\begin{array}{l}\text { Inpatient COVID-19 } \\
(n=34)\end{array}$ & $\begin{array}{l}\text { Inpatient comparators } \\
(n=102)\end{array}$ & $P$ value \\
\hline Female sex, $n(\%)$ & $27(79 \%)$ & $81(79 \%)$ & 1.00 \\
\hline Age in years, mean $[95 \% \mathrm{CI}]$ & $65.2[59.1-71.2]$ & $62.9[61.3-64.6]$ & 0.52 \\
\hline Race, $n(\%)$ & & & $0.92 *$ \\
\hline Caucasian & $9(26 \%)$ & $31(30 \%)$ & \\
\hline Black or African American & $22(65 \%)$ & $67(66 \%)$ & \\
\hline Othert & $3(9 \%)$ & $4(4 \%)$ & \\
\hline \multicolumn{4}{|l|}{ Comorbidities, $n(\%)$} \\
\hline Elixhauser index, mean $[95 \% \mathrm{CI}]$ & $10.2[6.8-13.5]$ & $9.5[8.6-10.3]$ & 0.72 \\
\hline Pulmonary disease & $8(24 \%)$ & $23(23 \%)$ & 1.00 \\
\hline Diabetes & $10(29 \%)$ & $30(29 \%)$ & 1.00 \\
\hline Renal disease & $12(35 \%)$ & $38(37 \%)$ & 1.00 \\
\hline Cancer & $2(6 \%)$ & $13(13 \%)$ & 0.36 \\
\hline Hypertension & $24(71 \%)$ & $76(75 \%)$ & 0.66 \\
\hline Coronary artery disease & $6(18 \%)$ & $17(17 \%)$ & 0.66 \\
\hline Congestive heart failure & $9(26 \%)$ & $34(33 \%)$ & 0.53 \\
\hline Obesity, BMI 30+ kg/m2 & $13(38 \%)$ & $32(31 \%)$ & 0.53 \\
\hline Smoking & $10(29 \%)$ & $38(37 \%)$ & 0.53 \\
\hline \multicolumn{4}{|l|}{ AI diagnosis, $n(\%)$} \\
\hline RA & $13(38 \%)$ & $38(37 \%)$ & 1.00 \\
\hline SLE & $5(15 \%)$ & $30(29 \%)$ & 0.11 \\
\hline PMR or GCA & $7(21 \%)$ & $7(7 \%)$ & 0.04 \\
\hline Sarcoidosis & $2(6 \%)$ & $5(5 \%)$ & 1.00 \\
\hline ILD & $2(6 \%)$ & $1(1 \%)$ & 0.15 \\
\hline Vasculitis & $2(6 \%)$ & $1(1 \%)$ & 0.15 \\
\hline Sjogren's syndrome & $1(3 \%)$ & $4(4 \%)$ & 1.00 \\
\hline MCTD & $1(3 \%)$ & $2(2 \%)$ & 1.00 \\
\hline Anti-synthetase syndrome & $0(0 \%)$ & $3(3 \%)$ & 0.57 \\
\hline Castleman disease/TAFRO & $1(3 \%)$ & $0(0 \%)$ & 0.25 \\
\hline Irritable bowel disease & $0(0 \%)$ & $1(1 \%)$ & 1.00 \\
\hline Inflammatory myopathy & $0(0 \%)$ & $1(1 \%)$ & 1.00 \\
\hline Inflammatory arthritis & $0(0 \%)$ & $1(1 \%)$ & 1.00 \\
\hline Mixed RA and SLE & $0(0 \%)$ & $4(4 \%)$ & 0.57 \\
\hline SLE, RA, and Sjogren's disease & $0(0 \%)$ & $1(1 \%)$ & 1.00 \\
\hline Scleroderma & $0(0 \%)$ & $1(1 \%)$ & 1.00 \\
\hline Takayasu arteritis & $0(0 \%)$ & $1(1 \%)$ & 1.00 \\
\hline \multicolumn{4}{|l|}{ Medications, $n(\%)$} \\
\hline Oral glucocorticoid & $24(71 \%)$ & $59(58 \%)$ & 0.23 \\
\hline$>10 \mathrm{mg} /$ day & $14(41 \%)$ & $38(38 \%)$ & 0.84 \\
\hline HCQ & $10(29 \%)$ & $52(51 \%)$ & 0.03 \\
\hline bDMARDs & $3(9 \%)$ & $11(11 \%)$ & 1.00 \\
\hline Rituximab & $3(9 \%)$ & $3(3 \%)$ & 0.16 \\
\hline anti-TNF & $0(0 \%)$ & $3(3 \%)$ & 0.57 \\
\hline Infliximab & $0(0 \%)$ & $2(2 \%)$ & 1.00 \\
\hline Tocilizumab & $0(0 \%)$ & $2(2 \%)$ & 1.00 \\
\hline Ustekinumab & $0(0 \%)$ & $1(1 \%)$ & 1.00 \\
\hline csDMARDs & $12(35 \%)$ & $43(42 \%)$ & 0.55 \\
\hline Methotrexate & $5(15 \%)$ & $20(20 \%)$ & 0.62 \\
\hline
\end{tabular}


Table 2 (continued)

\begin{tabular}{|c|c|c|c|}
\hline Characteristics & $\begin{array}{l}\text { Inpatient COVID-19 } \\
(n=34)\end{array}$ & $\begin{array}{l}\text { Inpatient comparators } \\
(n=102)\end{array}$ & $P$ value \\
\hline MMF & $4(12 \%)$ & $10(10 \%)$ & 0.75 \\
\hline Azathioprine & $1(3 \%)$ & $6(6 \%)$ & 0.68 \\
\hline Leflunomide & $2(6 \%)$ & $2(2 \%)$ & 0.26 \\
\hline Mesalamine/sulfasalazine & $0(0 \%)$ & $5(5 \%)$ & 0.33 \\
\hline tsDMARDs & $0(0 \%)$ & $1(1 \%)$ & 1.00 \\
\hline Tofacitinib & $0(0 \%)$ & $1(1 \%)$ & 1.00 \\
\hline Cyclophosphamide & $1(3 \%)$ & $0(0 \%)$ & 0.25 \\
\hline Sirolimus & $1(3 \%)$ & $0(0 \%)$ & 0.25 \\
\hline Vedolizumab & $0(0 \%)$ & $1(1 \%)$ & 1.00 \\
\hline Tacrolimus & $0(0 \%)$ & $1(1 \%)$ & 1.00 \\
\hline \multicolumn{4}{|l|}{ Presenting symptoms } \\
\hline Fever & $25(74 \%)$ & $16(16 \%)$ & 0.00 \\
\hline Cough & $24(71 \%)$ & $27(27 \%)$ & 0.00 \\
\hline Dyspnea & $18(53 \%)$ & $42(42 \%)$ & 0.17 \\
\hline Myalgias & $10(29 \%)$ & $1(1 \%)$ & 0.00 \\
\hline Diarrhea & $9(26 \%)$ & $12(12 \%)$ & 0.03 \\
\hline Loss of taste or smell & $5(15 \%)$ & $0(0 \%)$ & 0.00 \\
\hline \multicolumn{4}{|l|}{ Laboratory values, mean $[95 \% \mathrm{CI}]$} \\
\hline Creatinine & $2.35[1.03-3.68]$ & $2.64[1.99-3.29]$ & 0.69 \\
\hline C-reactive protein & 160.99 [117.35-204.63] & 76.09 [42.74-109.45] & 0.00 \\
\hline D Dimer & 3180.8 [1781.8-4579.7] & $4422.3[1788.1-7056.5]$ & 0.40 \\
\hline Ferritin & 1004.57 [346.69-1662.45] & $1406.48[469.94-2343.03]$ & 0.47 \\
\hline Lactate dehydrogenase & $466.15[305.40-626.90]$ & 408.71 [211.19-606.23] & 0.65 \\
\hline Absolute neutrophil count & $8.08[6.45-9.72]$ & $8.79[7.61-9.96]$ & 0.48 \\
\hline Absolute lymphocyte count & $1.51[1.24-1.78]$ & $1.48[1.31-1.64]$ & 0.86 \\
\hline Neutrophil-to-lymphocyte ratio & $10.58[7.54-13.62]$ & $9.65[7.68-11.62]$ & 0.61 \\
\hline White blood cell count & $12.06[9.53-14.58]$ & $12.47[11.04-13.90]$ & 0.77 \\
\hline Minimum albumin & 2.89 [2.69-3.09] & $3.17[3.05-3.29]$ & 0.02 \\
\hline \multicolumn{4}{|l|}{ Outcomes, $\mathrm{n}(\%)$ or mean $[95 \% \mathrm{CI}]$} \\
\hline Intensive Care Admission & $17(50 \%)$ & $27(26 \%)$ & 0.01 \\
\hline Intubation & $10(29 \%)$ & $6(6 \%)$ & 0.00 \\
\hline Days of intubation & $12.9[7.8-18.0]$ & $4.3[1.1-7.4]$ & 0.02 \\
\hline CRRT & $1(3 \%)$ & $2(2 \%)$ & 0.44 \\
\hline Days hospitalized & $12.9[9.4-16.3]$ & $8.0[5.5-10.4]$ & 0.03 \\
\hline Disch to home self-care & $14(41 \%)$ & $69(68 \%)$ & 0.01 \\
\hline Disch with home health & $8(24 \%)$ & $20(20 \%)$ & 0.63 \\
\hline Disch to rehabilitation & $3(9 \%)$ & $8(8 \%)$ & 1.00 \\
\hline Disch to hospice & $3(9 \%)$ & $3(3 \%)$ & 0.16 \\
\hline Patients re-hospitalized & $4(11 \%)$ & $30(29 \%)$ & 0.04 \\
\hline Death & $6(18 \%)$ & $3(3 \%)$ & 0.01 \\
\hline
\end{tabular}

*P value for Race was computed by merging "Caucasian" and "Other" race categories and comparing to "Black or African American"

† "Other" race includes American Indian or Alaska Native, Native Hawaiian or other Pacific Islander and not reported

* Pulmonary disease includes asthma or COPD

${ }^{\S} R A$, rheumatoid arthritis; $S L E$, systemic lupus erythematosus; $P M R$, polymyalgia rheumatica; $G C A$, giant cell arteritis; $I B D$, irritable bowel disease; $I L D$, interstitial lung disease; $M C T D$, mixed connective tissue disease; $b D M A R D s$, biologic disease modifying anti-rheumatic drugs; $T N F$, tumor necrosis factor; $c s D M A R D s$, conventional synthetic disease modifying anti-rheumatic drugs; tsDMARDs, targeted synthetic disease modifying antirheumatic drugs 
Table 3 Baseline characteristics and outcomes in rheumatic disease patients with COVID-19 who died ( $n=6)$ and survived ( $n=28)$ hospitalizations

\begin{tabular}{|c|c|c|c|}
\hline Characteristic & $\begin{array}{l}\text { COVID-19 Death } \\
(n=6)\end{array}$ & $\begin{array}{l}\text { COVID-19 Survive } \\
(n=28)\end{array}$ & $P$ value \\
\hline Female sex, $n(\%)$ & $4(67 \%)$ & $23(82 \%)$ & 0.58 \\
\hline Age in years, mean $[95 \% \mathrm{CI}]$ & $76.5[65.6-87.4]$ & $62.8[56.5-69.0]$ & 0.01 \\
\hline Race & & & $0.03 *$ \\
\hline Caucasian & $3(50 \%)$ & $6(21 \%)$ & \\
\hline Black or African American & $1(17 \%)$ & $21(75 \%)$ & \\
\hline Other $\dagger$ & $2(33 \%)$ & $1(4 \%)$ & \\
\hline \multicolumn{4}{|l|}{ Comorbidities, $n(\%)$} \\
\hline Elixhauser index, mean $[95 \% \mathrm{CI}]$ & $5.25(0 \%)$ & $10.96(0 \%)$ & 0.07 \\
\hline Pulmonary disease $\$$ & $0(0 \%)$ & $8(29 \%)$ & 0.30 \\
\hline Diabetes & $2(33 \%)$ & $8(29 \%)$ & 1.00 \\
\hline Renal disease & $0(0 \%)$ & $12(43 \%)$ & 0.07 \\
\hline Cancer & $0(0 \%)$ & $2(7 \%)$ & 1.00 \\
\hline Hypertension & $4(67 \%)$ & $20(71 \%)$ & 1.00 \\
\hline Coronary artery disease & $2(33 \%)$ & $4(14 \%)$ & 0.28 \\
\hline Congestive heart failure & $1(17 \%)$ & $8(29 \%)$ & 1.00 \\
\hline Obesity, BMI 30+ kg/m2 & $0(0 \%)$ & $13(46 \%)$ & 0.06 \\
\hline Smoking & $1(17 \%)$ & $9(32 \%)$ & 0.64 \\
\hline Former & $1(17 \%)$ & $9(32 \%)$ & \\
\hline Current & $0(0 \%)$ & $0(0 \%)$ & \\
\hline \multicolumn{4}{|l|}{ AI diagnosis, $n(\%)$} \\
\hline Rheumatoid arthritis & $3(50 \%)$ & $10(36 \%)$ & 0.65 \\
\hline SLE & $0(0 \%)$ & $5(18 \%)$ & 0.56 \\
\hline PMR or GCA & $2(33 \%)$ & $5(18 \%)$ & 0.58 \\
\hline Sarcoidosis & $0(0 \%)$ & $2(7 \%)$ & 1.00 \\
\hline Interstitial lung disease & $1(17 \%)$ & $1(4 \%)$ & 0.33 \\
\hline Vasculitis & $0(0 \%)$ & $2(7 \%)$ & 1.00 \\
\hline Sjogren's syndrome & $0(0 \%)$ & $1(4 \%)$ & 1.00 \\
\hline MCTD & $0(0 \%)$ & $1(4 \%)$ & 1.00 \\
\hline Castleman disease/TAFRO & $0(0 \%)$ & $1(4 \%)$ & 1.00 \\
\hline \multicolumn{4}{|l|}{ Medications, $n(\%)$} \\
\hline Oral glucocorticoid & $6(100 \%)$ & $18(64 \%)$ & 0.15 \\
\hline$>10 \mathrm{mg} /$ day & $5(83 \%)$ & $9(32 \%)$ & 0.06 \\
\hline Hydroxychloroquine & $1(17 \%)$ & $9(32 \%)$ & 0.64 \\
\hline bDMARDs & $1(17 \%)$ & $2(7 \%)$ & 0.45 \\
\hline Rituximab & $1(17 \%)$ & $2(7 \%)$ & 0.45 \\
\hline csDMARDs & $1(17 \%)$ & $11(39 \%)$ & 0.39 \\
\hline Methotrexate & $0(0 \%)$ & $5(18 \%)$ & 0.56 \\
\hline MMF & $1(17 \%)$ & $3(11 \%)$ & 0.56 \\
\hline Azathioprine & $0(0 \%)$ & $1(4 \%)$ & 1.00 \\
\hline Leflunomide & $0(0 \%)$ & $2(7 \%)$ & 1.00 \\
\hline Cyclophosphamide & $0(0 \%)$ & $1(4 \%)$ & 1.00 \\
\hline Sirolimus & $0(0 \%)$ & $1(4 \%)$ & 1.00 \\
\hline \multicolumn{4}{|l|}{ Presenting symptoms } \\
\hline Fever & $4(67 \%)$ & $21(75 \%)$ & 0.31 \\
\hline Cough & $3(50 \%)$ & $21(75 \%)$ & 0.64 \\
\hline Dyspnea & $2(33 \%)$ & $16(57 \%)$ & 1.00 \\
\hline Myalgias & $2(33 \%)$ & $8(29 \%)$ & 0.36 \\
\hline Diarrhea & $0(0 \%)$ & $9(32 \%)$ & 0.64 \\
\hline
\end{tabular}


Table 3 (continued)

\begin{tabular}{|c|c|c|c|}
\hline Characteristic & $\begin{array}{l}\text { COVID-19 Death } \\
(n=6)\end{array}$ & $\begin{array}{l}\text { COVID-19 Survive } \\
(n=28)\end{array}$ & $P$ value \\
\hline Loss of taste or smell & $0(0 \%)$ & $5(18 \%)$ & 1.00 \\
\hline \multicolumn{4}{|l|}{ Laboratory values } \\
\hline Creatinine & $1.64[0.78-2.50]$ & $2.50[0.02-3.25]$ & 0.32 \\
\hline C-reactive protein & $215.45[79.72-351.18]$ & 147.38 [168.01-262.89] & 0.28 \\
\hline D Dimer & $3402.2[-2262.3-9066.6]$ & 3123.0 [1995.8-4808.5] & 0.91 \\
\hline Ferritin & $1612.60[-947.52-4172.72]$ & 814.56 [962.23-2262.97] & 0.46 \\
\hline Lactate dehydrogenase & $456.50[282.78-630.22]$ & 468.90 [249.01-663.99] & 0.92 \\
\hline Absolute neutrophil count & $9.91[4.02-15.80]$ & $7.74[8.13-11.69]$ & 0.39 \\
\hline Absolute lymphocyte count & $1.21[0.60-1.81]$ & $1.56[0.90-1.52]$ & 0.22 \\
\hline Neutrophil-to-lymphocyte ratio & $9.26[4.54-13.98]$ & $10.82[5.67-12.85]$ & 0.53 \\
\hline White blood cell count & $19.60[9.88-29.32]$ & $10.44[8.22-12.66]$ & 0.07 \\
\hline Minimum albumin & $2.77[2.13-3.41]$ & $2.91[2.54-2.99]$ & 0.60 \\
\hline \multicolumn{4}{|l|}{ Outcomes, $n(\%)$ or mean $[95 \% \mathrm{CI}]$} \\
\hline Intensive Care Admission & $6(100 \%)$ & $11(39 \%)$ & 0.00 \\
\hline Intubation & $4(67 \%)$ & $6(21 \%)$ & 0.03 \\
\hline Days of intubation & $15.0[7.5-22.5]$ & $11.2[7.6-14.8]$ & 0.39 \\
\hline CRRT & $0(0 \%)$ & $1(4 \%)$ & 1.00 \\
\hline Days hospitalized & $18.7[10.0-27.4]$ & $11.6[15.0-22.4]$ & 0.19 \\
\hline
\end{tabular}

* $P$ value for Race was computed by merging "Caucasian" and "Other" race categories and comparing to "Black or African American"

$\dagger$ "Other" race includes American Indian or Alaska Native, Native Hawaiian or other Pacific Islander and not reported

ॠ Pulmonary disease includes asthma or COPD

$\S R A$, rheumatoid arthritis; $S L E$, systemic lupus erythematosus; $P M R$, polymyalgia rheumatica; $G C A$, giant cell arteritis; $I B D$, irritable bowel disease; $I L D$, interstitial lung disease; $M C T D$, mixed connective tissue disease; $b D M A R D s$, biologic disease modifying anti-rheumatic drugs; $c s D M A R D s$, conventional synthetic disease modifying anti-rheumatic drugs

hypertension, cardiovascular disease, lung disease, and diabetes, although this study has a significantly larger COVID-19 population $(n=600)$.

Other studies claim that TNF is involved in the proinflammatory activity of the cytokine storm that occurs in COVID-19 infection, leading to end-organ damage and poor patient outcomes. Therefore, it is hypothesized that anti$\mathrm{TNF} \alpha$ medications play protective roles in COVID-19 infection $[10,11]$. Another explanation for improved outcomes in patients using DMARDs is that these patients follow up with their rheumatologist more closely and have better control of their underlying disease [12]. Furthermore, these patients typically require less chronic steroid use which is a known risk factor for worse outcomes [3]. In our study, none of the ten COVID-19 patients taking anti-TNF $\alpha$ medications required hospitalization, while other DMARDs did not impact patient outcomes. In patients using rituximab, the other bDMARD included, three of five patients required hospitalization, and one patient died. Conventional synthetic DMARD (csDMARDs) use was also similar between those hospitalized and treated as outpatients. Therefore, this study supports that only anti-TNF $\alpha$ medications are protective in COVID-19 infection, rather than DMARD medications in general. Further studies including a quantitative measure of rheumatic disease control or activity may help to further distinguish reasons for patients having better outcomes on anti-TNF $\alpha$ medications.

Our results also add to the body of evidence suggesting that chronic glucocorticoid use increases disease severity, with glucocorticoids being used at nearly twice the frequency among hospitalized COVID-19 patients (71\% vs 36\%) [3]. Furthermore, of the six COVID-19 patients who died, all received chronic glucocorticoids, and five of these six used $10 \mathrm{mg} /$ day or more of prednisone. This reinforces that highdose chronic steroid use prior to COVID-19 infection is associated with worse outcomes [3]. We speculate that with a larger study population, risk of poor outcomes with chronic steroid use may be further stratified using daily dosing and treatment duration.

Our study is unique as we compared outcomes among rheumatic disease patients with COVID-19 infection to a matched group without COVID-19 infection. This provides an important clarification among those hospitalized with COVID-19, as admitted rheumatic disease patients have poor outcomes irrespective of infection [6]. By comparing our infected patients to comparators, this study clarifies the degree to which COVID-19 infection impacts hospitalization 
outcomes. The higher rate of ICU admission, ventilation, and death among COVID-19 patients is particularly concerning, as these results confirm the suspicion that rheumatic disease patients infected with COVID-19 are particularly susceptible to a severe disease course and do poorly clinically.

The statistically significantly lower minimum albumin concentrations and higher maximum CRP values seen in hospitalized COVID-19 patients are supported by other studies as increased CRP and decreased albumin have both been reported as prognostic indicators of disease progression and more severe COVID-19 infection [13].

One of our study's strengths is in comparing outcomes in a large healthcare system with a significantly diverse population. This study's racial is enriched with $65 \%$ African Americans, differing from similar studies looking at primarily Caucasian populations [4]. Appropriate racial representation is important as there is concern that African American populations face an excess disease burden [14]. Our study's overall hospitalization rate of $49 \%$ was slightly higher than those in similar studies with a hospitalization rate of $46 \%$ reported by Gianfrancesco and 44\% reported by D'Silva [3, 4]. Our rates of ICU admission were similar between our study and D'Silva's study (50\% vs $48 \%$, respectively). Furthermore, our $9 \%$ mortality rate matched the mortality rate reported by Gianfrancesco and was higher than the $6 \%$ mortality rate reported by D'Silva. However, in our study, the hospitalization rate among African Americans was similar to the overall hospitalization rate, and only one of six patients who died was African American. Aside from differing racial composition among these studies, factors such as comorbid and rheumatic disease burden may impact the difference in the observed outcomes. Unlike D'Silva and Gianfrancesco's studies, this study only includes patients actively taking immunosuppressive medications. This may result in a self-selection for a higher proportion of active rheumatic disease. In addition, none of these studies specifies the severity of comorbid conditions. Given that each of these populations shares different demographic backgrounds, severity of comorbid conditions among each of these patient populations is likely to differ and confound the outcome results. More than half of our patients are from African ancestry who are known to have more severe autoimmune conditions [15].

The strength of this study is that it is the first to evaluate the outcomes of rheumatic disease patients on active treatment for their conditions with immunosuppressive medication among a significantly diverse population with many African American patients. It is also the first to compare infected, admitted rheumatic disease patients with their uninfected rheumatic disease counterparts.

This study's power is limited by the sample size, especially when comparing COVID-19 patients who died or survived. Furthermore, we do not account for rheumatic conditions or comorbidity severity, and do not account for dosage and duration of medication regimens. These factors likely contribute to clinical course and outcomes. This study builds on the growing body of information on risk factors and clinical course of COVID-19 in rheumatic disease patients.

Supplementary Information The online version contains supplementary material available at https://doi.org/10.1007/s10067-021-05578-x.

Data availability Due to privacy and ethical concerns, the supporting data is not publicly available.

\section{Compliance with ethical standards}

Conflict of interest Dr. Khosroshahi reports grant from Pfizer and personal fees from Viela Bio, outside of the submitted work.

Ethics approval and consent to participate The study was determined to be exempt by the Emory University eIRB.

Code availability N/A.

\section{References}

1. Brito CA, Paiva JG, Pimentel FN, Guimarães RS, Moreira MR (2020) COVID-19 in patients with rheumatological diseases treated with anti-TNF. Ann Rheum Dis. https://doi.org/10.1136/ annrheumdis-2020-218171

2. Monti S, Balduzzi S, Delvino P, Bellis E, Quadrelli VS, Montecucco C (2020) Clinical course of COVID-19 in a series of patients with chronic arthritis treated with immunosuppressive targeted therapies. Ann Rheum Dis 79(5):667-668

3. Gianfrancesco M, Hyrich KL, Al-Adely S, Carmona L, Danila M, Gossec L et al (2020) Characteristics associated with hospitalisation for COVID-19 in people with rheumatic disease: data from the COVID-19 global rheumatology Alliance physician-reported registry. Ann Rheum Dis 79(7):859-866

4. D'Silva KM, Serling-Boyd N, Wallwork R, Hsu T, Fu X, Gravallese EM, Choi HK, Sparks JA, Wallace ZS (2020) Clinical characteristics and outcomes of patients with coronavirus disease 2019 (COVID-19) and rheumatic disease: a comparative cohort study from a US 'hot spot.'. Ann Rheum Dis 79(9):1156-1162

5. Zhao J, Pang R, Wu J, Guo Y, Yang Y, Zhang L, Xia X (2020) Clinical characteristics and outcomes of patients with COVID-19 and rheumatic disease in China 'hot spot' versus in US 'hot spot': similarities and differences. Ann Rheum Dis:annrheumdis-2020218183. https://doi.org/10.1136/annrheumdis-2020-218183

6. Feng X, Zou Y, Pan W, Wang X, Wu M, Zhang M et al (2011) Prognostic indicators of hospitalized patients with systemic lupus erythematosus: a large retrospective multicenter study in China. J Rheumatol 38(7):1289-1295

7. Janssen N, Karnad D, Guntupalli K (2002) Rheumatologic diseases in the intensive care unit: epidemiology, clinical approach, management, and outcome. Crit Care Clin 18(4):729-748

8. Zheng Z, Peng F, Xu B, Zhao J, Liu H, Peng J et al (2020) Risk factors of critical \& mortal COVID-19 cases: a systematic literature review and meta-analysis. J Inf Secur 81(2):e16-e25

9. Sharmeen S, Elghawy A, Zarlasht F, Yao Q (2020) COVID-19 in rheumatic disease patients on immunosuppressive agents. Semin Arthritis Rheum 50(4):680-686 
10. Li G, Fan Y, Lai Y, Han T, Li Z, Zhou P, Pan P, Wang W, Hu D, Liu X, Zhang Q, Wu J (2020) Coronavirus infections and immune responses. J Med Virol 92(4):424-432

11. Tay M, Poh C, Rénia L, MacAry P, Ng L (2020) The trinity of COVID-19: immunity, inflammation and intervention. Nat Rev Immunol 20:363-374

12. Ledingham J, Gullick N, Irving K, Gorodkin R, Aris M, Burke J, Gordon P, Christidis D, Galloway S, Hayes E, Jeffries A, Mercer S, Mooney J, van Leuven S, Galloway J, on behalf of the BSR and BHPR Standards, Guidelines and Audit Working Group (2017) BSR and BHPR guideline for the prescription and monitoring of non-biologic disease-modifying anti-rheumatic drugs. Rheumatology 56(6):865-868
13. Pourbagheri-Sigaroodi A, Bashash D, Fateh F, Abolghasemi H (2020) Laboratory findings in COVID-19 diagnosis and prognosis. Clin Chim Acta 510:475-482

14. Rentsch C, Kidwai-Khan F, Tate J, Park L, King J, Skanderson M (2020) Covid-19 by Race and ethnicity: a national cohort study of 6 million United States veterans. medRxiv. https://doi.org/10.1101/ 2020.05.12.20099135v1

15. Ramos PS, Shedlock AM, Langefeld CD (2015) Genetics of autoimmune diseases: insights from population genetics. J Hum Genet 60(11):657-664

Publisher's note Springer Nature remains neutral with regard to jurisdictional claims in published maps and institutional affiliations. 